\title{
Peculiar J-type Carbon Stars and Li
}

\author{
S. Lorenz-Martins \\ Observatório do Valongo/UFRJ, Ladeira Pedro Antonio, 43, Rio de \\ Janeiro, 20080-090 Brazil \\ N. A. Drake \\ Observatório Nacional/MCT, R. José Cristino, 77, Rio de Janeiro, \\ 20921-400 Brazil \\ Astronomical Institute of St. Petersburg State University, St. Petersburg,
198904 Russia
}

\begin{abstract}
We determined the carbon and lithium abundances and carbon isotopic ratios for 4 peculiar carbon stars by means of spectral synthesis method. Li abundances were derived using $\mathrm{Li}$ resonance line at $\lambda 6707.8 \AA$. For carbon abundance determinations we used the lines of the red system of $\mathrm{CN}$ molecule. Spectral region of $\lambda \lambda 7994-8030 \AA$ containing the lines of molecules ${ }^{12} \mathrm{CN}$ and ${ }^{13} \mathrm{CN}$ was used also for ${ }^{12} \mathrm{C} /{ }^{13} \mathrm{C}$ ratio measuring. The high-resolution spectra were obtained on the $1.52 \mathrm{~m}$ telescope of ESO with the Fiber-fed Extended Range Optical Spectrograph (FEROS). These observations were obtained under the agreement between the CNPq-Observatório Nacional, Brazil, and ESO.
\end{abstract}

\section{Introduction}

The evolution along the AGB and third dredge-up transform an oxygen-rich giant in a carbon-rich star enhanced with $s$-process elements and ${ }^{12} \mathrm{C}$. However, J-type carbon stars appear not to evolve in the same way as ordinary carbon stars, they have ${ }^{12} \mathrm{C} /{ }^{13} \mathrm{C}$ ratio about 4 and a lack of $s$-process elements. Furthermore, they present a very strong Li line at $\lambda 6707 \AA$. These stars, like the carbon-rich stars, usually present the characteristic emission in the infrared at $11.3 \mu \mathrm{m}$ due to $\mathrm{SiC}$ grains. However, this emission is not present in some J-type carbon stars (Little-Marenin 1986). These special stars, named Peculiar J-type carbon stars, present emission at $9.7 \mu \mathrm{m}$, which is due to silicate grains. This detection indicates that the circumstellar envelopes of peculiar J-type stars should be oxygen-rich, on the contrary to the photospheric chemical compositions characterized by $\mathrm{C} / \mathrm{O}>1$.

\section{Observations}

The high-resolution spectra of our sample were obtained on the $1.52 \mathrm{~m}$ telescope of ESO with the spectrograph FEROS in December 1998. The wavelength cov- 
erage in one exposure (object $=$ sky) is $\lambda \lambda 3600-9200 \AA$, with resolving power of $\lambda / \Delta \lambda=48000$. The $\mathrm{S} / \mathrm{N}$ ratio was about 80 .

\section{Results and Conclusions}

Since their discovery, carbon stars with silicate emission have been suggested to be J-type carbon stars. However, ${ }^{12} \mathrm{C} /{ }^{13} \mathrm{C}$ ratios have been determined only for a very small number of peculiar J-type stars. In this work we determined for the first time the carbon isotopic ratios for 3 carbon stars with oxygen shells: C 749, C 1003 and C 1130. BM Gem, a well known J-type peculiar star was used for comparison. Synthetic spectra were calculated using the last version of the MOOG program (Sneden 1973). The molecular line lists for studied regions were taken from Drake (1998). Atmospheric models of Johnson (1982) calculated for superficial gravity of $\log g=0.0$ were used for all stars under consideration. We determined also the radial velocities of the stars. In Table 1 we present the results obtained for our sample.

Table 1. Peculiar J-type C stars

\begin{tabular}{ccccrcccc}
\hline Star & $T_{\text {eff }}$ & {$[\mathrm{C}]$} & {$[\mathrm{N}]$} & {$[\mathrm{O}]$} & $\mathrm{C} / \mathrm{O}$ & $\log \epsilon(\mathrm{Li})$ & ${ }^{12} \mathrm{C} /{ }^{13} \mathrm{C}$ & $\begin{array}{c}V_{\text {rad }} \\
\mathrm{km} / \mathrm{s}\end{array}$ \\
\hline BM Gem & 3000 & 0.36 & -0.30 & -0.01 & 1.002 & 1.2 & 10 & 82.8 \\
C 749 & 2500 & 0.07 & -0.70 & -0.30 & 1.002 & 0.8 & 5 & 99.2 \\
C 1003 & 3000 & 0.37 & -0.30 & 0.00 & 1.003 & 1.0 & 8 & 38.0 \\
C 1130 & 3000 & 0.37 & -0.30 & 0.00 & 1.003 & 0.8 & 30 & 38.7 \\
\hline \hline
\end{tabular}

Our analysis showed that C 749 is a high velocity middle metal-poor star $([\mathrm{Fe} / \mathrm{H}]=-0.5)$, which seems indicate that it belongs to the thick disk stars. As concerned C 1130, we have found that it is not a J-type carbon star; its isotopic ratio is typical for ordinary carbon stars.

Although our sample is small, it seems that we have found an anti-correlation between $\mathrm{Li}$ and ${ }^{13} \mathrm{C}$ abundances. In addition, the $\mathrm{C} / \mathrm{O}$ ratios are very close to unity, which seems indicate that these stars are very close to SC stars, in a transition phase between oxygen and carbon-rich stars. The idea of a transition scenario was proposed by several authors (e.g. Lorenz-Martins 1996). Meantime, it is necessary to take into account a larger sample.

Acknowledgments. S.L.M. and N.A.D. thank FAPERJ for financial support under the contracts E-26/150.726/99 and E-26/151.172/98 respectively.

\section{References}

Drake, N.A. 1998, PhD Thesis, Observatorio Nacional, Rio de Janeiro

Johnson, H. 1982, ApJ, 260, 254

Little-Marenin, I. 1986, ApJ, 307, L15

Lorenz-Martins, S. 1996, A\&A, 314, 209

Sneden, C. 1973, A current version of the LTE line analysis code MOOG 